\title{
Nanocrystalline Materials and Nanometer-Sized Glasses
}

\author{
H. Gleiter, Saarbrücken
}

(University of the Saarland and Institute of New Materials)

Nanocrystalline materials are single or multiphase polycrystals, of crystal size in the order of a few $(1-10) \mathrm{nm}$, whose existence and potential significance were pointed out less than a decade ago [1]. At present, these materials are most frequently generated by high-pressure consolidation of small randomly oriented crystals with clean surfaces. Nanocrystalline materials which have also been called nanophase materials, ultra-fine grain sized materials, ultra-fine microstructures and nanometer-sized materials, are of interest for the following four reasons:

(1) Nanocrystalline materials seem to exhibit an atomic structure which differs from that of the two known solid states: the crystalline (with long range order) and the glassy (with short range order). They comprise a new (third) state with a statistical atomic structure $[2,3,4]$.

(2) The properties of nanocrystalline materials differ (in some cases by several orders of magnitude) from those of glasses and/or crystals with the same chemical composition, which suggests that they may be utilized technologically in the future.

(3) Nanocrystalline materials seem to permit the alloying of conventionally immiscible components such as metals and ceramics. The resulting alloys have been observed to exhibit new and technologically attractive properties.

(4) If small (1-10 $\mathrm{nm}$ diameter) glass droplets are consolidated (instead of small crystals), a new type of glass, called nanometer-sized glass, is obtained. Such glasses differ structurally from conventional glasses in the sense that their short range order extends to next nearest neighbours only. The atomic arrangement for interatomic spacings beyond the shell of next nearest neighbours seems random.

\section{Basic Ideas}

A schematic cross-section through a hard sphere model of a nanocrystalline material is shown in Fig. 1, wherein all atoms are assumed to be chemically identical. Structurally, two kinds may be distinguished: "crystal atoms", the nearest neighbour configurations of which correspond to the lattice configuration, and "boundary atoms" characterized by nearest neighbour configurations which are different. Hence, in these terms a nanocrystalline material consists of a crystalline component (formed by all the crystal atoms) and a boundary component with a froth-like morphology (formed by all the boundary atoms). For a single component material, the atomic structure of all crystals is identical, e.g. all crystals in nanocrystalline Fe have a b.c.c. lattice. However, the atomic structures of the various boundaries are different because their atomic arrangement depends, among other parameters, on the orientation relationship between the adjacent crystals. If the crystals are crystallographically oriented at random, the relationship between the crystals 1 and 2 (Fig. 1) is different from that between 2 and 3. Hence, the atomic arrangement in the boundary $A$ differs from the one in $B$. In other words, the interatomic spacings (atomic matching) between the boundary atoms forming boundary $A$ (e.g. the spacings $a b, a c)$ differ from the spacings between the atoms forming boundary B (e.g. the spacings de, df).

The same applies to all other boundaries. As nanocrystalline materials contain typically about $10^{19}$ boundaries per $\mathrm{cm}^{3}$, the interfacial component represents the sum of over $10^{19}$ atomic arrangements all of which are different. If there is no correlation in the sense that specific interatomic spacings do not occur preferentially in many of the boundaries, the interfacial component represents a structure which exhibits no short-range order. This does not imply that grain boundaries are disordered. Indeed, every boundary is assumed to have a two-dimensionally ordered structure. However, the periodicity of this structure and thus the interatomic spacings within every boundary differ from boundary to boundary. The physical reason for this new type of structure is the constraint imposed on the atoms in the cores of the boundaries by the adjacent crystal lattices of different orientations.

Similar arguments seem to apply to the contact region between small particles with a glassy structure leading

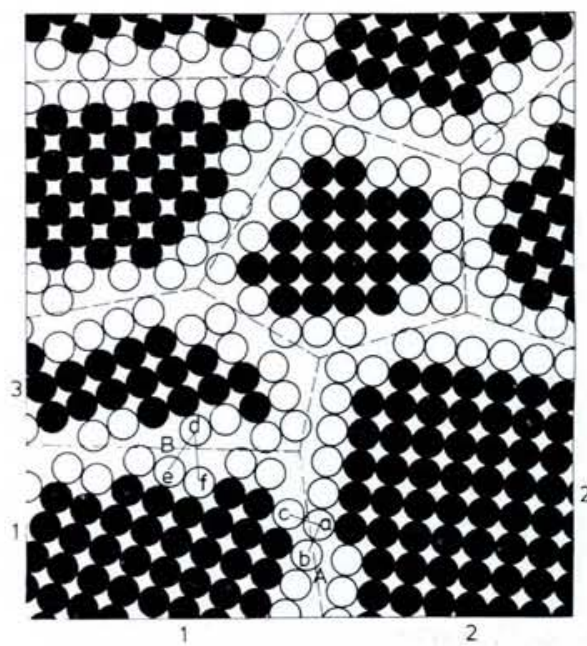

Fig. 1 - Schematic (two-dimensional) representation of a nanocrystalline material distinguishing between the atoms associated with the individual crystals (filled circles) and those constituting the boundary network (open circles). All crystals are assumed to have the same atomic arrangement. The boundary atoms are shown in regular lattice positions, but in reality will relax to form different atomic arrangements. The atoms in the boundaries and in the crystals are assumed to be chemically identical.

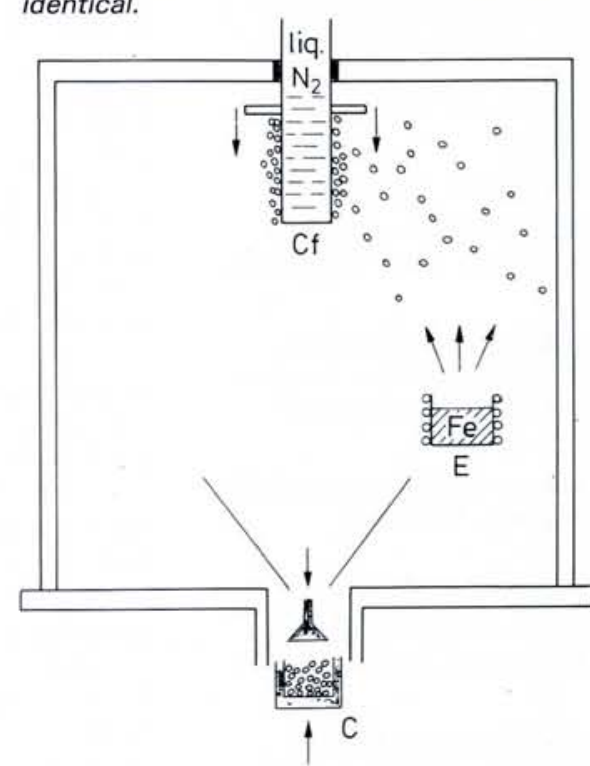

Fig. 2 - Experimental arrangement used to generate nanocrystalline materials by the inert gas condensation method. E represents an evaporator (containing $\mathrm{Fe}$ ), $\mathrm{C}_{f}$ is a cold finger held at liquid nitrogen temperature. $C=$ consolidation device. 


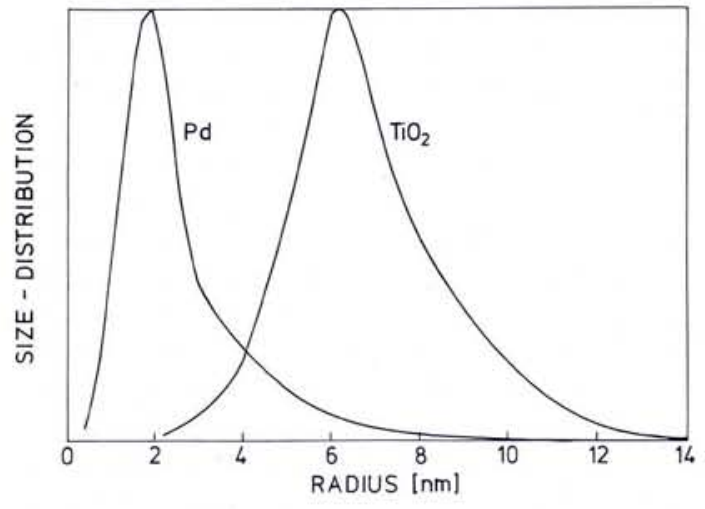

Fig. 3 - Distribution of the crystal sizes in nanocrystalline $\mathrm{TiO}_{2}$ measured by transmission electron microscopy, and nanocrystalline Pd, measured by small angle neutron diffraction.

to the formation of nanometer-scale glasses (q.v.).

\section{Preparation of Nanocrystalline Materials}

The experimental arrangement used most frequently to prepare nanocrystalline materials is a modified gas condensation method (Fig. 2), where the material (e.g. Fe) is evaporated into an inert gas atmosphere (e.g. He, pressure about $1 \mathrm{kPa}$ ). Through interatomic collisions with the $\mathrm{He}$ atoms, the evaporated $\mathrm{Fe}$ atoms lose kinetic energy and condense in the form of small crystals a few nanometers across which accumulate on a vertical cold finger as a loose powder. After restoring high vacuum $1<$ $10^{-6} \mathrm{~Pa}$ ) the powder is stripped off and funnelled into a piston and anvil device where it is compacted (pressure up to 5 $\mathrm{GPa}$ ). Sputtering, electron gun or laser evaporation may be employed instead of thermal evaporation.

Other methods which have been utilized to obtain fine grained powders suitable for generating nanocrystalline materials are ball milling (applied to Ru and AIRu), vapour-phase synthesis (applied to various ceramics and semiconductors) and precipitation from solutions or gels. In these last two cases special care has to be taken to remove surface contaminants.

A systematic study of the impurity content of nanocrystalline materials

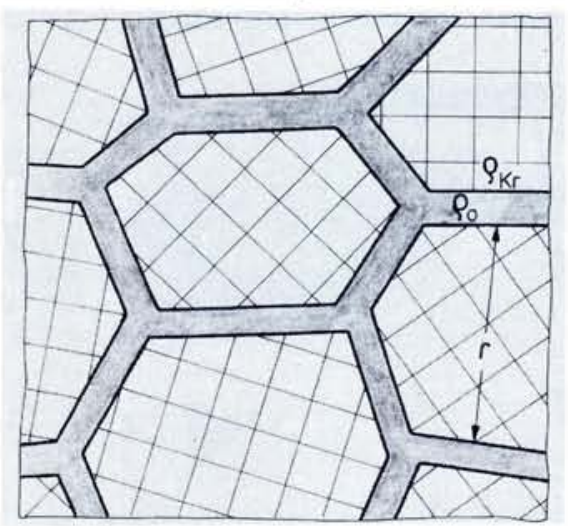

Fig. 4 - Schematic drawing of the microstructure of a nanocrystalline material. prepared by inert gas condensation revealed that gaseous and metallic impurities were less than $0.1 \%$ and $\mathrm{He}$ less than $50 \mathrm{ppm}$. The density of metallic nanocrystalline materials varied between $85 \%$ and $90 \%$ of the crystalline density (depending on the material) approaching $100 \%$ after grain growth. The distribution of crystal sizes in Pd and $\mathrm{TiO}_{2}$ determined by transmission electron microscopy and small angle $\mathrm{X}$-ray or neutron scattering is presented in Fig. 3.

\section{Microstructure}

Studies of the microstructure of nanocrystalline materials have been carried out by means of transmission electron microscopy, X-ray diffraction, neutron diffraction and EXAFS. The microstructure deduced is summarized in Fig. 4. Crystals (average diameter $r$, density $\rho_{\mathrm{Kr}_{\mathrm{r}}}$ ) are separated by grain boundaries of thickness about $1 \mathrm{~nm}$ and density $\left(\rho_{0}\right)$ some $50 \%$ of the crystal density and hence considerably lower than in glasses. This low density of the boundary region seems to be crucial for the atomic structure and many of the properties of nanocrystalline materials.

\section{Atomic Structure}

The atomic structure has been studied by $\mathrm{X}$-ray diffraction and EXAFS. The former studies were performed by comparing the measured interference function of a nanocrystalline material with the one computed by assuming the atoms in the boundaries to be either statistically arranged or in a structure corresponding to a glass. In the computations, the crystal size, the size distribution, the boundary density, the boundary thickness, etc. were matched to the experimental data.

As many be seen from Fig. $5 a$, the diffraction curve predicted by assuming a random atomic arrangement in the boundaries agrees well with the measurements, but not if the boundary regions are assumed to have a glassy structure (Fig. 5b).
The same conclusion was obtained from the EXAFS measurements. These indicated that nanocrystalline materials comprise two structural components: one (representing the crystallites) in which the atoms are arranged in the same pattern as in the bulk crystal, and a second (representing the average over all boundaries) in which the atomic arrangement is random. In other words, a structure which may be regarded as a "frozen-in gas" in the same sense as a glass is a "frozen-in liquid". Clearly, such a random (gas-like) arrangement is only possible if all kinds of interatomic spacing exist, and this implies a reduced atomic density in the interfaces as has been observed.

If nanocrystalline materials contain a new type of atomic structure, not only should the diffraction properties be modified, but evidence should also come from methods and structure sensitive

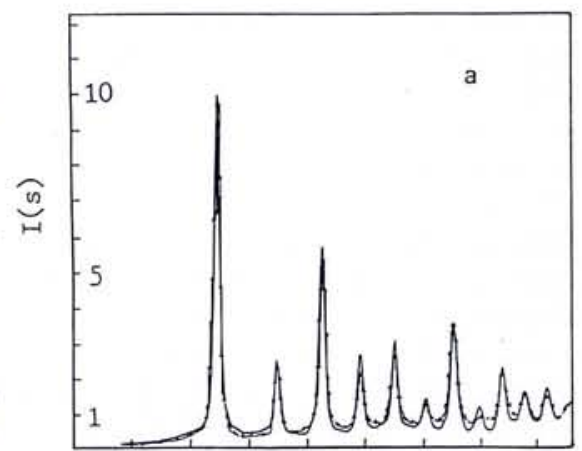

$\begin{array}{llllllllll}0 & 0.2 & 0.4 & 0.6 & 0.8 & 1.0 & 1.2 & 1.4 & 1.6 & \times 10\end{array}$

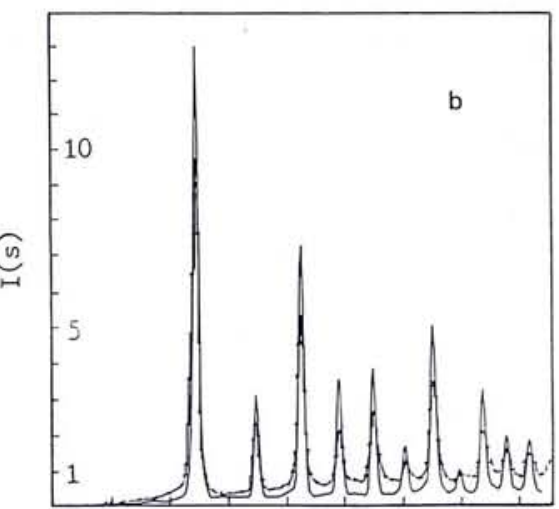

$\begin{array}{llllllllll}0 & 0.2 & 0.4 & 0.6 & 0.8 & 1.0 & 1.2 & 1.4 & 1.6 \times 10\end{array}$ wave vector $2 \sin \theta / \lambda\left[\mathrm{nm}^{-1}\right]$

Fig. 5 - Comparison of the measured $(+-+-+)$ and computed $(\longrightarrow)$ interference functions of nanocrystalline $\mathrm{Fe}(6 \mathrm{~nm}$ average crystal size). The model system assumed for the computations is a boundary structure consisting of four atomic layers in which:

(a) atoms are statistically ("gas-like") arranged. The boundary thickness of four atomic layers agrees with the results of the small angle neutron diffraction and hydrogen solubility measurements.

(b) atoms form a short-range ordered (glasslike) structure similar to the one measured in $\mathrm{Fe}_{80} \mathrm{~B}_{20}$. 
Table I - Comparison between the properties of materials in the nanocrystalline, glassy and crystalline state

\begin{tabular}{|l|c|c|c|c|c|}
\hline Properties & Unit & Material & $\begin{array}{c}\text { Nanocrystalline } \\
\text { state }(5-10 \mathrm{~nm})\end{array}$ & $\begin{array}{c}\text { Glassy } \\
\text { state }\end{array}$ & $\begin{array}{c}\text { Crystalline } \\
\text { state }\end{array}$ \\
\hline $\begin{array}{l}\text { Self diffusion } \\
\text { Solute diffusion } \\
(239 \mathrm{~K})\end{array}$ & $\mathrm{m}^{2} / \mathrm{s}$ & $\begin{array}{c}\mathrm{Cu} \\
\mathrm{Ag} \text { in Cu }\end{array}$ & $2 \times 10^{-19}$ & $2 \times 10^{-40}$ \\
\hline $\begin{array}{l}\text { Plasticity } \\
(293 \ldots 495 \mathrm{~K})\end{array}$ & $10^{-6} \mathrm{~K}^{-1}$ & $\mathrm{Cu}$ & 36 & 18 & 17 \\
\hline $\begin{array}{l}\text { Thermal expan- } \\
\text { sion (300 K) }\end{array}$ & $\mathrm{J} / \mathrm{g} \times \mathrm{K}$ & $\mathrm{Pd}$ & 0.38 & 0.26 & 0.25 \\
\hline $\begin{array}{l}\text { Specific heat } \\
(150 \ldots 300 \mathrm{~K})\end{array}$ & $\mathrm{CaF} \mathrm{F}_{2}$ & ductile & & $0.001 \%$ \\
\hline $\begin{array}{l}\text { Solute solubility } \\
(373 \mathrm{~K})\end{array}$ & $\mathrm{Bin} \mathrm{Cu}$ & $4 \%$ & 215 & 222 \\
\hline $\begin{array}{l}\text { Saturation } \\
\text { magnetization } \\
(4 \mathrm{~K})\end{array}$ & $\mathrm{Fe}$ & 130 & & \\
\hline
\end{tabular}

properties. In practice, Mössbauer and positron lifetime spectroscopy as well as measurements of the specific heat, the entropy, the thermal expansion, diffusivity and the enhanced solute solubility all seem to be consistent with the new atomic structure $[2,3,4]$.

The Gibbs Free Energy $(\triangle G)$ of a nanocrystalline material relative to a single crystal of the same chemical composition has been determined by comparing their specific heats, $\Delta c_{\mathrm{p}}$, and the electromotive force, emf. The result for $\mathrm{Pd}$ between $\mathrm{O}$ and $323 \mathrm{~K}$ is shown in Fig. 6. The negative slope, $-\delta G / \delta T$, of this curve is the excess entropy $(\triangle S)$ of nanocrystalline $\mathrm{Pd}$ relative

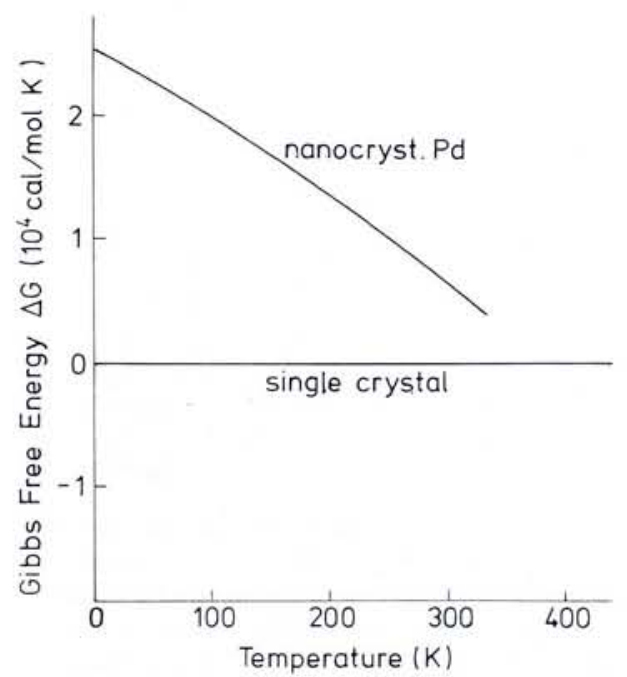

Fig. 6 - Gibbs Free Energy $(\triangle G)$ of nanocrystalline $P d(8 \mathrm{~nm}$ crystal size) relative to a Pd single crystal (horizontal line at $\Delta G=0$ ) as a function of temperature deduced from $\Delta c_{p}$ and emf measurements. In the case of nanocrystalline $\mathrm{Cu}$, similar results were obtained; the $\triangle G$-curve of the nanocrystalline $\mathrm{Cu}$ was found to intersect the single crystal curve at about $350 \mathrm{~K}$. to a Pd single crystal. $\Delta S$ is found to be about $7 k_{\mathrm{B}}$ ( $k_{\mathrm{B}}$ is Boltzmann's constant) which is comparable to the entropy of evaporation of $\mathrm{Pd}$ (about $10 k_{\mathrm{B}}$ ) and almost an order of magnitude larger than the entropy of fusion (about $1 k_{\mathrm{B}}$ ). In other words, the large excess entropy agrees with the random ("gas-like") atomic structure deduced from the $X$ ray diffraction and EXAFS data.

\section{Properties}

Table I lists properties of some of the nanocrystalline materials investigated compared with those of the crystalline and glassy states of the chemically identical substance.

Typically, the properties of the crystalline and glassy states differ by about $5 \%$ or less, whereas the nanocrystalline state varies between $50 \%$ and a factor of up to $10^{21}$. Differences of this type are hard to understand unless the atomic structure is assumed to be different. Moreover the electronic structure also seems to vary, e.g. the Hall constant of nanocrystalline $\mathrm{Cu}$ was found to be about twice that of conventional $\mathrm{Cu}$.

Figs $7 \mathrm{a}$ and $7 \mathrm{~b}$ show examples of the ductile deformation observed in nano-
Fig. $7 a-$ Brittle fracture of conventional $\mathrm{TiO}_{2}$ ceramic and ductile behaviour of nanocrystalline $\mathrm{TiO}_{2}$ during indentation at $20^{\circ} \mathrm{C}$ [6]. crystalline ceramics. This and the enhanced diffusivity of nanocrystalline ceramics may be of interest for technological applications. The observed plasticity [6] seems to be based on grain boundary sliding and diffusional flow. As both deformation modes are possible in any material, any conventionally brittle material: ceramics, intermetallics or covalent substances (diamond, $\mathrm{Si}$, $\mathrm{Ge}$ ) can be expected to become ductile if generated in the nanocrystalline form.

Activation energies of diffusion are among the lowest measured so far in solids. Comparable figures have been reported only for fast ionic conductors, fast metallic diffusors and for $\mathrm{H}$ in some metals. Diffusion of $\mathrm{Ag}$ in nanocrystalline $\mathrm{Cu}$ (Fig. 8) occurs at the remarkably low level of $0.3 \mathrm{eV}$ and $0.64 \mathrm{eV}$ respectively in the temperature ranges $303-$ $343 \mathrm{~K}$ and $353-373 \mathrm{~K}$. For comparison, the activation energies of volume and grain boundary diffusion in the same system are $2 \mathrm{eV}$ and $1 \mathrm{eV}$, so that an enhancement of about 20 orders of magnitude relative to volume diffusion results.

\section{Nanometer-sized Glasses}

By analogy to nanocrystalline materials, the generation of new types of glass called "nanometer-sized glasses" is proposed. Their production may be carried out by a procedure similar to that described in Fig. 2. If the material evaporated has the ability to form a glass, the evaporation and subsequent rapid cooling in the $\mathrm{He}$ atmosphere may result in the formation of nanometersized glass droplets rather than crystallites. Consolidation may result in solids containing a high density of defects arising from the mechanical shear of the droplets during compaction or in the region of contact between adjacent particles. The contact region may differ structurally and/or chemically from that in the centre for the following reason. The surface atoms of an isolated glassy droplet in vacuum form a glassy atomic

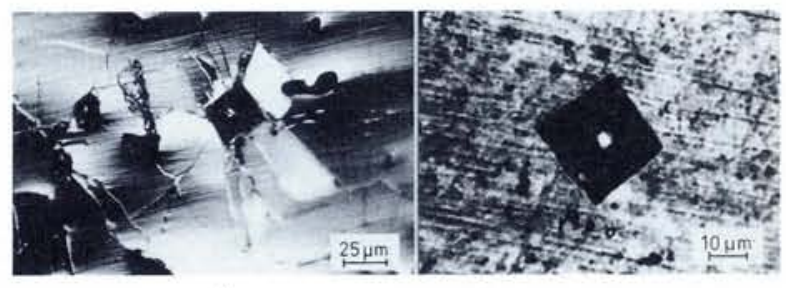

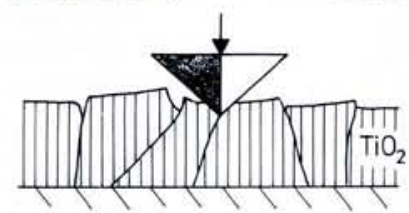

conv. $\mathrm{TiO}_{2}$ - ceramic $(50 \mu \mathrm{m})$

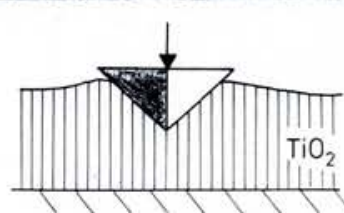

nanocryst. $\mathrm{TiO}_{2}(7 \mathrm{~nm})$ 


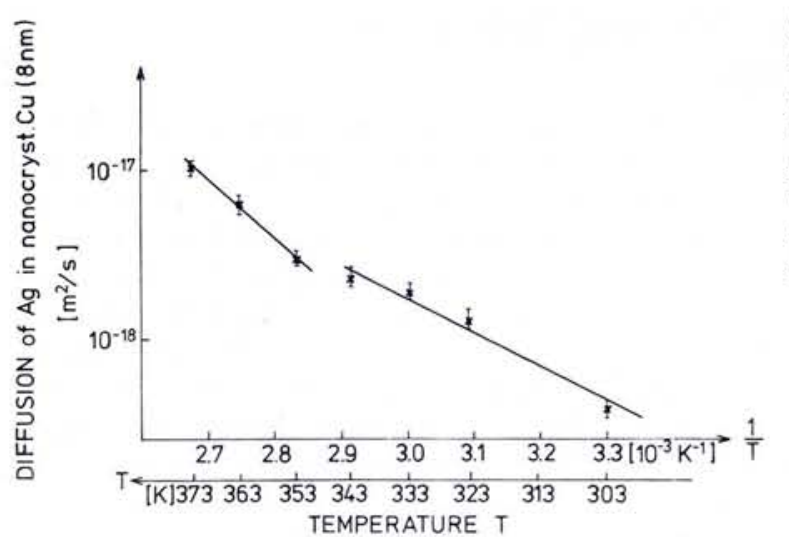

arrangement with those in the interior so that certain interatomic spacings are preferred. If two such droplets are brought into contact, the interatomic spacings between the surface atoms may then be different and although some relaxational motion may occur, a broader distribution of interatomic spacings in the interfacial region gives rise to a structure comparable to a bulk glass with the same chemical composition.

Similar arguments may apply to a region in which a glass droplet is mechanically sheared during compaction. Because of the non-periodic structure, a region of enhanced free volume results, as is known from the shear bands in metallic glasses. By analogy to the structure of nanocrystalline materials, the atomic structure of the interfacial regions of a nanometer-sized glass is proposed to be random. This speculation agrees with recent $\mathrm{X}$-ray diffraction and Mössbauer spectroscopy measurements $[7,8]$. In practice, the total atomic distribution function (Fig. 9a) of a nanometer-sized glass observed experimentally differs from that of a conventional glass (Fig. 9b). This may be understood by assuming that the nanometer-sized glass is formed by small centres with a glassy structure comprising next nearest neighbours only. These regions are arranged randomly relative to one another, otherwise higher order correlations would be ob-

Fig. $7 \mathrm{~b}$ - Plastic flow of nanocrystalline $\mathrm{CaF}_{2}$ at $80^{\circ} \mathrm{C}$. On the left is indicated the experimental arrangement used [6].
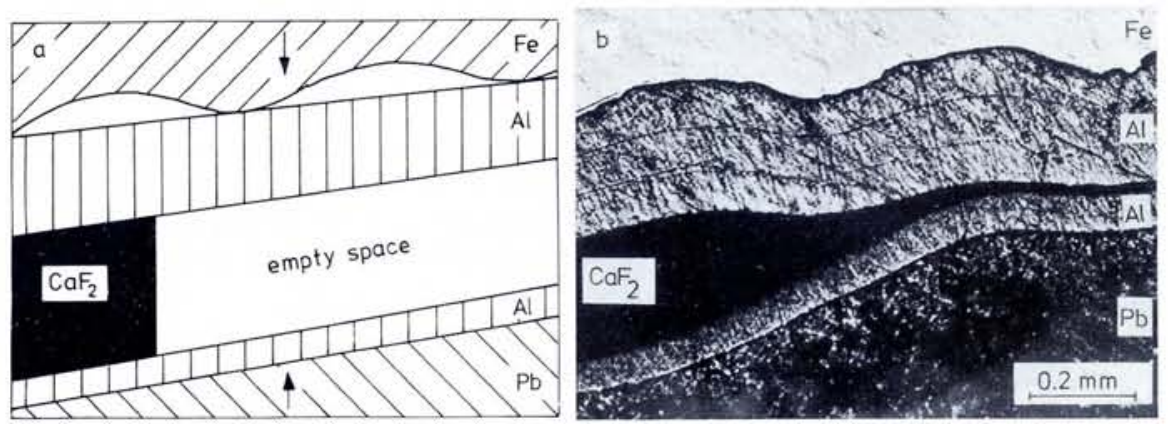

\section{REFERENCES}

- Diffusion coefficient of Ag in nanocrystalline $\mathrm{Cu}(8$ $\mathrm{nm}$ crystal size) as a function of temperature. From the slope of both curves, the activation energy of the diffusion process is deduced to be about $0.6 \mathrm{eV}$ and $0.3 \mathrm{eV}$.

served in the atomic distribution function. By varying the diameter and/or chemical composition of the consolidated glass droplets, the relative volume fractions of the glassy and the randomly structured component can be manipulated. In other words, by varying the size of the glassy particles used for consolidation, one can vary (at consistent chemical composition) the atomic structure of nanometer-sized glasses continuously between the short-range ordered structure of a conventional glass and an almost random atomic arrangement. If the chemical composition of the surface region of an isolated glass droplet differs from the central region (e.g. due to surface segregation effects), the interfacial regions deviate chemically as well as structurally from the centre.

Presumably, nanometer-sized glasses may not only be generated by evaporation and consolidation, but also by heavily deforming (e.g. by ball milling) bulk pieces of glass or by precipitation, atomizing or sputtering processes. It would seem that the way is open to a new type of glassy material.

\section{Acknowledgement}

This work has been supported by the BMFT under contract No. $523-400303 \mathrm{M} 234$ and the Deutsche Forschungsgemeinschaft (DFG). The co-operation with colleagues from the Universities of München, Saarbrücken and Stuttgart as well as from DESY Hamburg and ILL Grenoble, France, is appreciated.
[1] Gleiter H., Proceed. Second Risø Int. Symp. on Metallurgy and Materials Science, Eds. N. Hansen, A. Horsewell, T. Leffers, H. Lilholt (Ris $ø$ National Laboratory, Roskilde) 1981, p. 15.

[2] Birringer R. and Gleiter H., Advances in Materials Science, Encyclopedia of Mat. Sci. and Eng., Ed. R.W. Cahn (Pergamon Press, Oxford) 1988, p. 339.

[3] Siegel R.W. and Hahn H., Current Trends in Physics of Materials, Ed. M. Yusouff (World Scientific Publ. Co., Singapore) 1987 , p. 403.

[4] Schaefer H.E., Würschum R., Birringer R. and Gleiter H., J. Less-Common Metals 140 (1988) 161.

[5] Rupp J. and Birringer R., Phys. Rev. B 36 (1987) 7888.

[6] Karch J., Birringer R. and Gleiter H., Nature 330 (1987) 556.

[7] Weissmüller J., Birringer R. and Gleiter H., to be published.

[8] Jing J., Birringer R., Gonser U. and Gleiter $H_{\text {., }}$, to be published.
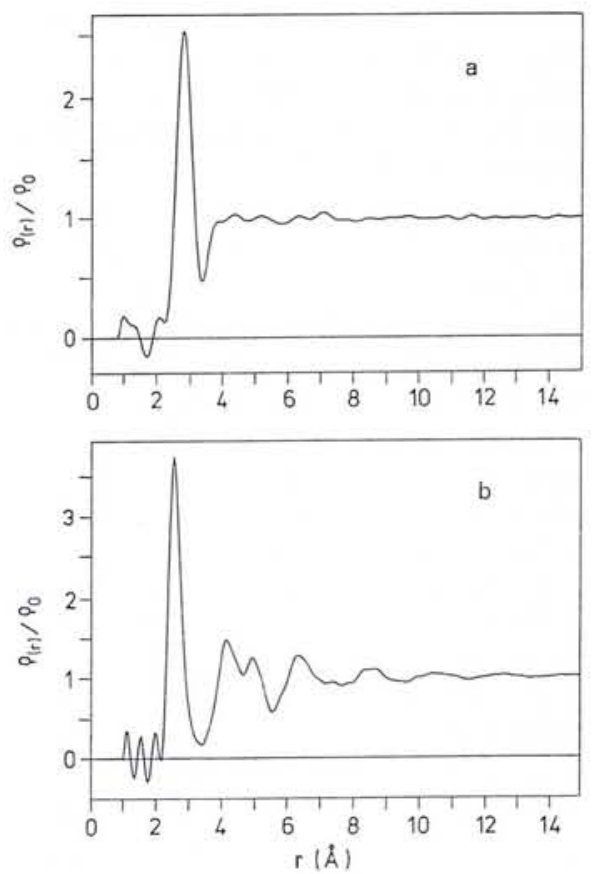

Fig. 9 - a) Total atomic distribution function of a nanometer-sized metallic glass, $A u_{75} \mathrm{Si}_{25}$. In the case of a statistical atomic structure $\rho(r) / \rho_{o}=1$. According to the measured atomic distribution function, nanometer-sized glasses resemble a random arrangement of atomic clusters. Each cluster consists of a central atom surrounded by a shell of nearest neighbours; $r$ is the interatomic spacing.

b) Total atomic distribution function, of a bulk $P_{24} F_{78} S_{9} i_{9} B_{13}$ metallic glass. $\rho(r) / \rho_{0}$ describes the relative probability of finding another atom in the vicinity of a reference atom located at $r=0 . \rho(r) / \rho_{0}$ averages over both types of atoms ( $\mathrm{Fe}$ and $\mathrm{P}$ ). Obviously, certain interatomic spacings, indicated by the maxima of $\rho(r) / \rho_{0}$ are preferred; $r$ is the interatomic spacing. 